\title{
Towards a low metallicity carbon star spectral library
}

\author{
R. Wahlin ${ }^{1}$, L. Mattsson ${ }^{1}$, S. Höfner ${ }^{1}$ and B. Aringer ${ }^{2}$ \\ ${ }^{1}$ Department of Astronomy and Space Physics, Uppsala University, \\ Box 515, SE-751 20 Uppsala Sweden \\ email: rurik.wahlin@astro.uu.se \\ ${ }^{2}$ Department of Astronomy, University of Vienna, \\ 1180 Wien, Türkenschanzstrae 17, Austria \\ email: aringer@astro.univie.ac.at
}

\begin{abstract}
It is well established that mass loss from AGB stars due to dust driven winds cannot be arbitrarily low. We model the mass loss from carbon rich AGB stars using detailed frequency-dependent radiation hydrodynamics including dust formation. We present a study of the thresholds for the mass loss rate as a function of stellar parameters based on a subset of a larger grid of such models and compare these results to previous theoretical work. Furthermore, we demonstrate the impact of the pulsation mechanism and dust formation for the creation of a stellar wind and how it affects these thresholds and briefly discuss the consequences for stellar evolution.
\end{abstract}

Keywords. stars: AGB and post-AGB, atmospheres, mass loss

\section{The need for a synthetic spectral library}

AGB stars dominate the infrared spectra of intermediate age populations (?). This has also been studied more recently by e.g. ?? and ?. The fraction of AGB stars that at a given time are carbon stars increases with decreasing metallicity (e.g. ?, and references therein) and at low metallicities carbon stars are more numerous than M-type AGB stars.

Luminous AGB stars are long period variables. This has two main consequences when synthesising spectra for stellar populations. First, observed spectral libraries would require a large number of spectra to cover the pulsational period. Second, hydrostatic model atmospheres cannot be used to reproduce all features in the spectra. Some spectral features (e.g. molecular absorption due to $\mathrm{CN}$ and $\mathrm{C}_{2}$ ) are very similar in synthetic spectra in the hydrostatic approximation and the corresponding time averaged synthetic spectra from hydrodynamical model atmospheres. Other features (e.g. the absorption features of $\mathrm{HCN}$ and $\mathrm{C}_{2} \mathrm{H}_{2}$ ) are strongly affected by the inclusion of hydrodynamics in the model atmospheres, see figure 1(a).

The synthetic spectra are now fairly good and ? find that spectra from present model atmospheres can reproduce observed spectra of carbon stars in a large wavelength range.

\section{Models and spectra}

The spectra are computed from spherically symmetric hydrodynamic model atmospheres computed with frequency dependent radiative transfer (???).

Opacities for the synthetic spectra were computed with the COMA code (?) Atomic opacities were taken from from VALD (?), molecular opacities were taken from several sources and are constantly being updated. The diatomic molecules included in the calculations were: $\mathrm{CO}, \mathrm{CN}, \mathrm{C}_{2}, \mathrm{CH}, \mathrm{HF}, \mathrm{HCl}, \mathrm{FeH}$ and $\mathrm{CrH}$. The polyatomic molecules 

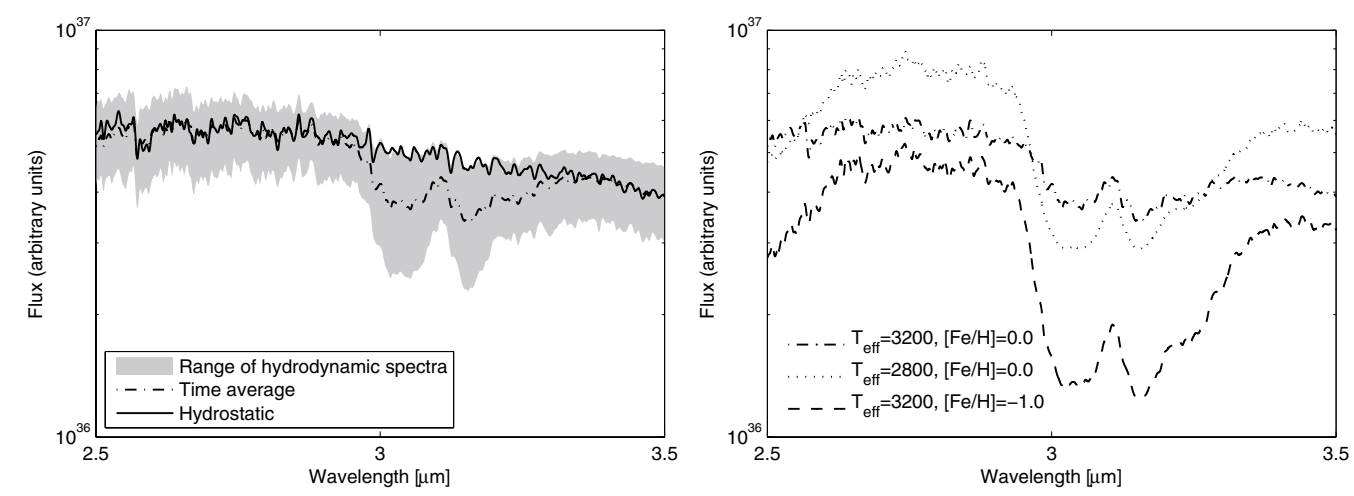

Figure 1. Left (a): The 3 micron band due to $\mathrm{HCN}$ and $\mathrm{C}_{2} \mathrm{H}_{2}$ which is formed in the outer part of the stellar atmospheres is significantly different between the time-averaged hydrodynamic and hydrostatic synthetic spectra. Other features (e.g. due to $\mathrm{CN}_{2}$ and $\mathrm{CH}$ ) are quite similar in the hydrostatic and hydrodynamic cases. Right (b): The strength of the 3 micron band is strongly dependent on several stellar parameters. Here we show how the strengths are affected when varying $\mathrm{T}_{\text {eff }}$ and $[\mathrm{Fe} / \mathrm{H}]$ while keeping other stellar parameters constant.

included were: $\mathrm{HCN}, \mathrm{C}_{3}$ and $\mathrm{C}_{2} \mathrm{H}_{2}$. Opacities from amorphous carbon were also included in the calculations.

A low metallicity grid of hydrodynamic model atmospheres with the corresponding time averaged synthetic spectra is presently being computed. It is crucial to let the spectral library cover all stellar parameters that will affect the spectra. In figure $1(b)$ we show the effect on the synthetic spectra of varying the effective temperature or the overall metallicity keeping other stellar parameters constant. The grid coverage will be $2500 \leqslant T_{\text {eff }} \leqslant 3500,7.0 \leqslant \log (C-O) \leqslant 9.1,5000 \leqslant L_{*} \leqslant 10000,0.5 M_{\odot} \leqslant M_{*} \leqslant 4 M_{\odot}$ and $-2 \leqslant[\mathrm{Fe} / \mathrm{H}] \leqslant 0$. 